\title{
Biomedical technology and the clinic of the future
}

\author{
Technology pioneers trade views with a clinician and an entrepreneur on the likely impact of large-scale systems \\ technology in healthcare.
}

$\Gamma_{\mathrm{m}}^{\mathrm{o}}$ o date, large-scale 'omics data sets and systems approaches in biology have had a relatively minor impact on the practice of medicine. As new technology brings individual genome sequencing closer to reality and large-scale biology continues to progress, opportunities are likely to open up in disease prediction, prevention, diagnosis and treatment. Here the views of two researchers on the potential of disruptive biomedical technologies in clinical practice are contrasted with the perspectives of a clinician and an entrepreneur in commercial clinical information technology.
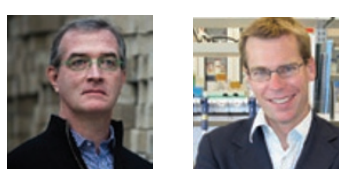

\section{POINT: Are we prepared for the future doctor visit?}

Stephen H Friend \& Trey Ideker

Imagine the following visit to the doctor's office, which, although fictitious, is based on technologies that are emerging or already available. A patient, Jane Doe, enters the clinic for a routine physical exam. Today, at least seven parameters would be registered upon her admittance: sex, age, height, weight, temperature, pulse rate and blood pressure (itself a pair of values). But in the future when Jane registers, this set of routine measurements will have expanded enormously (Table 1).

\section{Tomorrow's routine checkup}

Either on this visit or a previous one, Jane's full genome has been sequenced, noninvasively, using a buccal swab. At the same time, and optionally on every visit, the nurse has sampled and sequenced the metagenome of

Stephen H. Friend is at Sage Bionetworks, Seattle, Washington, USA. Trey Ideker is in the Departments of Medicine and Bioengineering, University of California, La Jolla, California, USA, and at The Institute for Genomic Medicine, University of California, La Jolla, California, USA. e-mail:friend@sagebase.orgor tideker@ucsd.edu the microbiome pool resident in the patient's mucosal and gastrointestinal cavities, providing a detailed characterization of the population of microbes commensal with the human host. Messenger RNA, microRNA, proteome and metabolome profiles may be gathered from urine and, if necessary, whole blood and other tissues. Finally, in addition to height and weight, a large panel of physiological parameters and images is monitored, capturing detailed information about respiration, endocrine function, cardiac and brain activity, and so on.

Another key development that will transform Jane's visit to the clinic is deeper data integration. All of the newly gathered information are banked in a unified electronic medical record, which uses a relational database to establish cross-references among the different data types. The new information augments the history of data gathered on previous visits, including all medical treatments and outcomes accumulated over the patient's lifetime.

Crucially, the new data are then integrated with a library of biological network models spanning multiple levels and scales (Fig. 1). First is the network of functional and molecular interactions-a.k.a. the molecular wiring diagram-providing a modular, hierarchical and executable view ${ }^{1}$ of the cellular processes underlying human health and disease. Such networks are being assembled from diverse large- and small-scale experiments performed over decades of systems biology and biomedical research, providing an up-to-date representation of current knowledge in the field ${ }^{2,3}$. A second type of network model will represent the relevant nosology, which maps relationships between diseases based on their similarities in etiology, pathogenesis and symptoms. Related to this will be another network-that of pharmacologic treatments, which provides rich information about the different protocols and drugs that are available along with their quantitative inter-relationships. One more important network will be the patient's extended social network and pedigree, which will be available along with references to the integrated medical records of friends and relatives. This social network documents significant personal relationships in Jane's life, weighted by importance and, subject to privacy concerns, gathered from social networking websites, personal address books, geographical co-location data, as well as cell phone and e-mail usage. The pedigree provides a complementary set of social relationships that have a genetic basis.

The benefits of these network models to Jane are severalfold. First, they integrate an array of different lines of evidence for health or disease, enabling the formulation of compound biomarkers that are combinations or functions of many simultaneous readouts. Such compound biomarkers can be more robust than biomarkers based on individual genes, proteins or metabolites ${ }^{4}$. Second, the networks provide a natural interpretation of the mechanisms behind Jane's present and future conditions, in contrast to current biomarkers that often have little relation to the actual cause of disease. Third, Jane's data and outcomes can be dynamically analyzed and reintegrated to 